\title{
Challenges for Teaching Wireless Communications Standards at the Graduate Level
}

\author{
Laura Pierucci $(\mathbb{D}$ \\ Department of Information Engineering, University of Florence, Via di Santa Marta 3, 50139 Firenze, Italy; \\ laura.pierucci@unifi.it
}

Received: 17 November 2019; Accepted: 11 December 2019; Published: 15 December 2019

\begin{abstract}
Telecom operators and companies ask for graduates with a specific education on the standards of the Institute of Electrical and Electronic Engineers (IEEE) or the $3^{\text {rd }}$ Generation Partnership Project (3GPP), and the University curricula must consider these needs. The standards are written in a technical form, in a language understandable only by experts, and the technical details and algorithms are not often outlined. Therefore, a new educational methodology must be applied because the teachers have to bridge the gap between the basic knowledge (and the poor technical language) of students and the technical specifics of the standards. The paper presents a structured methodology to provide innovative teaching of the wireless standards for the Engineering Master's degree, according to the Conceive, Design, Implement, and Operate (CDIO) initiative and project based learning. The methodology is organized in three learning phases to understand the standardization process and improve students' skills to implement standard compliant communications systems. This challenge can be only won with laboratory activities to assist students in understanding wireless standards and with hands-on experiences during the internship period at telecom operators with the vision of a close cooperation between universities and telecom operators. Only in this way can the students achieve a solid background in designing and developing prototypes compliant with wireless communications standards and working skills for their future professional engineering careers. The effectiveness of the adopted educational methodology to provide innovative learning of wireless standards is evaluated by questionnaires filled in online by students and by the achieved skills implemented as confirmed by telecom operators. In this vision, the paper provides decision support to leaders in educational organizations to teach wireless standards effectively.
\end{abstract}

Keywords: teaching wireless communications standards; 3GPP standards; Wi-Fi; LTE system; 5G system; wireless sensor networks; education and training

\section{Introduction}

The need for services at a high data rate and the massive number of devices (about nine billion mobile subscriptions are expected in 2022) able to connect different Radio Access Technologies (RATs) present new challenges for the future $5 \mathrm{G}$ systems [1,2]. Different generations of mobile systems (3G, 4G, and 5G), Wi-Fi, Bluetooth, and wireless sensor networks work together in a heterogeneous networks scenario. In the context of the Internet of Things (IoT), many interconnected end-user terminals, sensors, actuators, up to communications among vehicles (Machine-to-Machine (M2M)), and direct Device-to-Device (D2D) communications must guarantee the Quality of Services (QoS) requirements to end-users and meet their satisfaction with the services, the so-called Quality of Experience, (QoE) [3].

These standardization processes evolve very quickly in the telecommunication field, and experts are often highly specialized in a single issue of a particular standard. On the other hand, academicians have a broader knowledge, but with a more top level vision of the problem. It is mandatory to integrate 
education on standards into University curricula to bridge the gap between the theoretical knowledge of traditional courses and the new educational needs [4,5].

The paper presents a methodology based on the Conceive, Design, Implement, and Operate (CDIO) initiative [6] and Project Based Learning (PBL) [7] to improve the learning of wireless standards and understanding the constraints, complexity, and performance of standard specific implementation in real systems.

Our methodology is based on three sequential phases: (1) lectures to describe the standard, provided by the teacher; (2) the design and implementation of some building blocks of the subsystem/system selected as a case study according to standard specifications, with the support of tutors; (3) analysis and reporting of the implemented system's performance and the difficulties encountered in implementing the process, provided by the students.

The above methodological phases are applied to the course on Wireless Networks at the graduate level, which covers different wireless communications standards such as mobile systems (Universal Mobile Telecommunications System (UMTS) and Long Term Evolution (LTE), up to the Fifth-Generation (5G)), the IEEE 802.11 family for Wireless Local Area Networks (WLAN), Bluetooth, and the IEEE 802.15.4 standards for Wireless Personal Area Networks (WPAN).

Therefore, in the Wireless Networks course, the traditional lectures are jointly integrated with practical laboratory experiences and project based works developed at the laboratories of the University and the telecom operators. This structured and sequential methodology is fundamental in wireless standard teaching as lectures consolidate and improve the theoretical background of students and laboratory experience allows acquiring the technical and soft skills needed to implement a standard compliant real system.

A collaborative learning approach and a close collaboration with telecom providers can help better address the second phase of our methodology.

During the laboratory activities, the students can work in groups in a collaborative learning way and acquire skills to identify and combine different parameters for achieving specific results and testing the system. The students acquire the experience of working in a team, an important requirement in the working world. The same standards are defined by various stakeholders participating in different working groups, which propose methods, algorithms, and typical parameter values to implement and test a real system.

A close collaboration with industries and telecom operators via the internship period, laboratory experiences, and project based work provides to the students hands-on skills, which are one of the most relevant aspects in learning the standards. The standards focus on the implementation of a real system (or a product), and the industries and telecom operators have industrial projects to develop it. Therefore, the curriculum in Telecommunications Engineering includes a period of internship under the double coordination of the University and industry. The student can access the professional world, often for the first time, and explore new methods and tools, increasing the opportunities to enter into the labor market. Conversely, the industries have new graduates already able to use their system tools, to implement complex systems, to participate in standardization consortia, and propose new specifics or proprietary standards eventually.

Summarizing, the main challenges to improve standardization learning are: (1) the development of practical experiences and project work in laboratories; (2) collaborations with telecom operators by means of internships and participation in the working group of projects; (3) the use of a dynamic learning environment.

The remainder of the paper is organized as follows: Section 2 highlights the problems in teaching wireless communications standards. Section 3 includes the syllabus of the Wireless Networks course, the adopted methodology, the challenges, and the improvements for more effective learning of the standards. Section 4 shows the laboratory experiences, which comprise a valid approach to increase the students' interests in the wireless standards. Finally, the conclusions report the main items highlighted in the paper to improve the quality of standardization education at the Master's degree level. 


\section{Problems in Teaching Wireless Communications Standards}

Companies and research institutions are mainly involved in the wireless standardization process. The standards are often written as technical reports, specifications by telecommunications experts, and business participants in a complex, concise form [5]. Theoretical concepts, algorithms, and parameters are considered well known (as for the experts in the field); therefore, it is very difficult for the students to read and study directly the releases or series of standards. For example, the 3GPP TR36.814 v9.00 "Further advancements for E-UTRAphysical layer aspects" document of the LTE system specifies the uplink and downlink transmission schemes for spatial multiplexing and transmit diversity with different multiple antenna configurations. The reader should be already familiar with the characteristics and technical advantages of the different MIMO techniques to understand this specification.

Therefore, the teacher has to teach (i) first of all, the basis of MIMO techniques, explaining that the transmit diversity technique improves the reliability (i.e., bit error rate performance) by combating the fading multipath and that it is useful for delay sensitive services, while the MIMO spatial multiplexing methods achieve higher peak data rates by enabling multiple data stream transmissions between the eNodeB and the User Equipment (UE), and (ii) then, he/she can analyze the MIMO schemes considered by 3GPP Series 36 with the students.

Another problem is related to the updates of the successive versions of standards' specifications, referring often to other documents, increasing the difficulty in tracking and reading. For example, the phrase in 3GPP TR 36.814 v9.00 on page 12 Control signaling is multiplexed with data on PUSCH according to the same principle as in Rel-8" requires remembering or reading about uplink multiple access in Release 8.

Another difficulty in reading the standards series derives from the large use of acronyms, even if a list of acronyms is reported in all the documents.

Moreover, in the teaching of a course based on wireless communications standards, the teacher needs continuous updating and training of knowledge (i) to follow the evolution of standards, e.g., the standards for the mobile systems $2 \mathrm{G}, 3 \mathrm{G}, 4 \mathrm{G}$, up to the new $5 \mathrm{G}$ cellular systems, (ii) to analyze the different characteristics of several wireless systems such as cellular systems, Wi-Fi, Bluetooth, and ZigBee, and (iii) to understand the differences of the releases of the same standard.

For example, considering the evolution of the 3GPP standards (up to 2015), some releases introduced new network system architectures and air interface technologies such as in Release 8 (2009), where the Long Term Evolution (LTE) system was defined. In this all-IP network architecture, the circuit switching domain is not present with respect to Release 99 (Wideband Code Division Multiple Access (WCDMA) -Universal Mobile Telecommunication System (UMTS)). In Release 8, the Orthogonal Frequency Division Multiple Access/Single Carrier-Frequency Division Multiple Access (OFDMA/SC-FDMA) techniques are adopted, while UMTS uses Code Division Multiple Access (CDMA) as the air interface technology. Other releases do not modify fully the system architecture, but consider the improvements of performance with new methods such as the use of adaptive modulation and coding (in Release 5), carrier aggregation, and Multiple-Input Multiple-Output (MIMO) antenna technologies (in Release 7 and Release 13), and other releases address new aspects such as Cooperative Multipoint Transmission (CoMP), Heterogeneous Networks (HetNet) improvements (in Release 11), Machine Type Communications (MTC) (in Release 13), or D2D communications under cellular networks. Another example can be the family of IEEE 802.11 (first version in 1997) with the successive versions such as IEEE 802.11a, IEEE 802.11b, IEEE802.11g, and IEEE802.11n to improve the data rate in the $2.4 \mathrm{GHz}$ and $5.4 \mathrm{GHz}$ bandwidths. The security aspects areconsidered in the IEEE 802.11i version, and other versions signed with letters (e, f, k, p, r, s, $\mathrm{T}, \mathrm{u}, \mathrm{v}$ ) concern the extensions of the basic services and improvements of services already available, such as IEEE802.11e for QoS or IEEE 802.11p for wireless ability in vehicular environments.

An important aspect to highlight in teaching of standards is the use of radio frequency spectrum resources in the different countries and the adoption of different regulations. For example, the IEEE 
802.11a on the $5.4 \mathrm{GHz}$ bandwidth can be used in the U.S. without a license according to Part 15 of the FCC Rules and Regulations, while in Europe, this is not allowed as it can create interferences with radar and satellite services operating in this bandwidth. Therefore, the $802.11 \mathrm{~h}$ protocol was introduced in 2004 to satisfy the requirements of the ETSI EN 301893 regulation by using DFS (Dynamic Frequency Selection) and TPC (Transmit Power Control) techniques.

It is also important to inform the students of the different standardization activities of the main actors such as IEEE, 3GPP, and 3GPP2, e.g., the activities of IEEE starting from IEEE 802.11a based on OFDM have developed the IEEE $802.16 \mathrm{~m}$ system based on OFDMA, while LTE was derived from the experiences of $3 \mathrm{GPP}$ in the previous $3 \mathrm{G}$ systems.

\section{Methodology for Teaching Wireless Standards}

Due to the large impact of the standardization process on the economy and society, future professionals have to have skills to implement systems compliant with the wireless standards. The Wireless Networks course enclosed in the University curricula trains students in applying telecommunications standards. The course is structured in the second didactical period from February to June for a global number of $72 \mathrm{~h}$ providing 9 university training credits (CFU) based on ECTS (European Credit Transfer and Accumulation System) in the first year of the Master's degree (Master's of Science according to the European-Bologna Process 1999) in Telecommunication Engineering.

\subsection{Syllabus}

The author has taught Wireless Networks course since 2006 until now for the Master's degree in Telecommunications Engineering. During such a long period, the syllabus of the course has changed many times according to the evolution of wireless communications; for example, in the case of cellular systems, the first course analyzed the Global System for Mobile communications (GSM) standard, and now, it is only explained as a historical note. Currently, the syllabus covers the cellular systems, from UMTS, LTE, and LTE-Advanced, up to the new 5G system, as well as the main standards for the consumer market of WLAN and WPAN networks. The Wireless Networks course considers mainly the physical and MAC layers of each standard and also the use of new technologies, e.g., MIMO antenna systems to improve system capacity. For example, the physical and MAC layers for low power devices are shown to be compliant with the IEEE 802.15.4 standard, and also, the activities of ZigBee Alliance related to the upper layers are described such as security services, compliance testing, and marketing towards smart energy and home automation.

The curriculum of the Telecommunications Engineering Master's degree considers a number of basic courses, which must be completed before attending the Wireless Networks course, as in the following:

- Digital signal processing

- Digital communications techniques

- Information theory and coding

- Fundamentals of telecommunications networks

These preparatory courses provide the main theoretical knowledge for understanding wireless communications, which are considered as well known consequently in the Wireless Networks course. For example, the teacher explains the adoption of OFDMA in the LTE downlink, its advantage with respect to WCDMA, and the motivation for this choice, but she does not teach the OFDM modulation again.

\subsection{Methodology}

Educational methodologies based only on lectures, as in Science, Technology, Engineering, Mathematics, and Computer science (STEM-C) university programs, assure an effective theoretical background [8]. Unfortunately, they do not address the practical implementation oriented aspect of the 
standardization processes $[9,10]$. Therefore, lecture based education has to be joined with laboratory experiences [11] and project based work according to the CDIO initiative and PBL, to develop student's practical engineering skills, team awareness, and project management abilities.

According to this vision, the experiences in the laboratory and participation in the working project integrate traditional lectures into the Wireless Networks course, assuring highly qualified skills are gained by the future graduates, both in the real-world industry and in the participation in developing new standardization processes of private consortia or standardization organizations.

The adopted methodology for the course is organized into three sequential phases to achieve the specific learning objectives:

1. First, the teacher provides lectures of the considered standard recommendations. Teaching materials are loaded on the University's web platform. Standards documents and links are also shown to the students to understand, e.g., how the 3GPP series and working groups are organized.

2. Then, a simplified project is assigned to a group of students on a particular topic, and the student team designs the building blocks of the system by identifying the functionalities and the necessary parameters with the teacher's/tutor's supervision.

3. Finally, the students test the implemented system in terms of performance with respect to the specifications. A report written by the students reviews the performance analysis and the encountered difficulties to achieve the objectives.

The students can report their feedback by filling in a questionnaire to evaluate the effectiveness of this methodology of teaching in terms of the teacher's clarity during the lectures, the availability of materials, interest towards the explained topics, and the laboratories' utility. The questions are shown in Table 1, and the relative score assigned by the students reports an average score of eight in a range of [1-10] [12] for the Wireless Networks course, as shown in Figure 1.

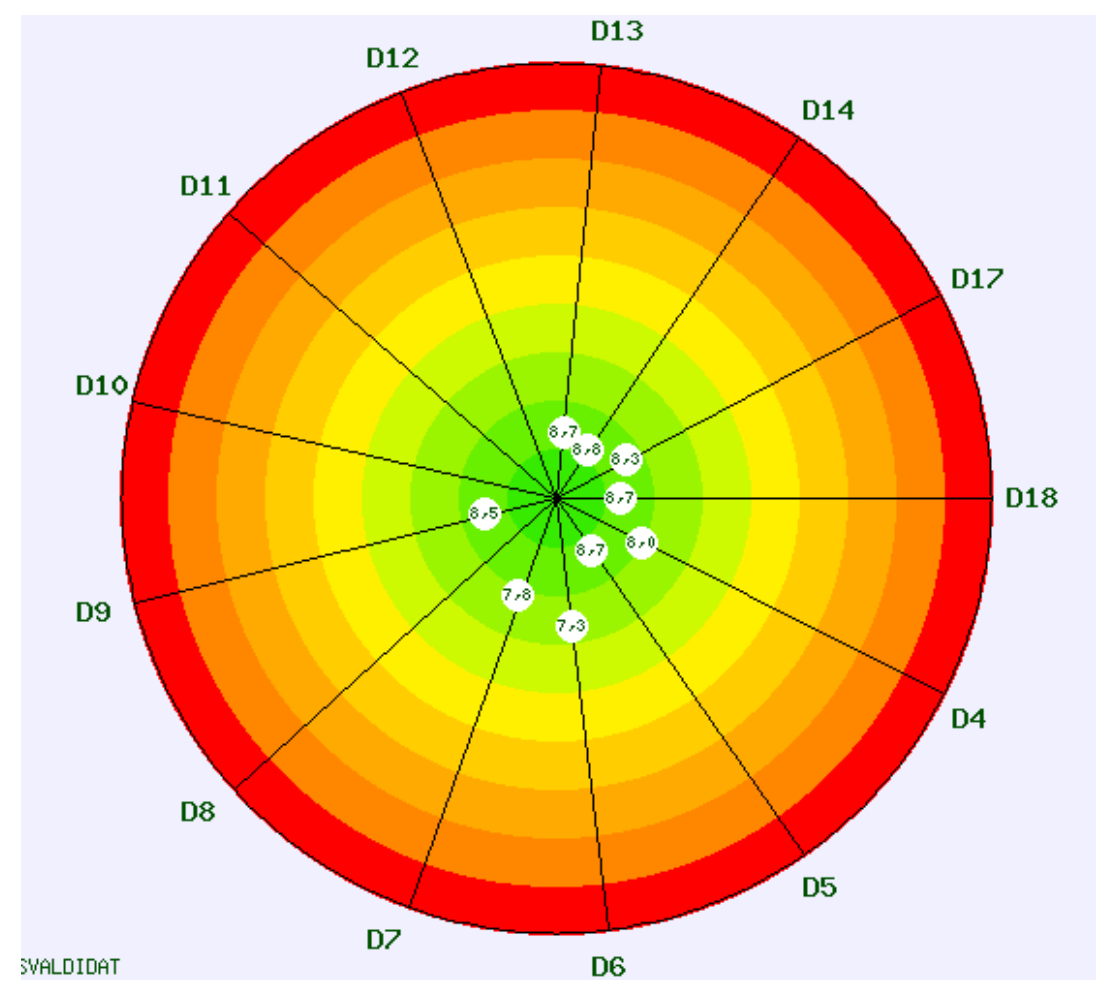

Figure 1. Scores related to the questions in Table 1. 
Table 1. Students' Questionair.

\begin{tabular}{cl}
\hline Questions & \\
\hline D4: & Is the previous basic knowledge sufficient for understanding the topics covered? \\
D5: & Are the topics of the course new or supplementary compared to the knowledge already \\
D6: & Is the study load of this teaching proportionate to the credits awarded? \\
D7: & Is the teaching material (indicated or provided) adequate for the study \\
D9: & Have the examination procedures been clearly defined? \\
D13: & Are educational activities (exercises, laboratory experiences, workshops, seminars, etc.) useful \\
D14: & Is the teacher available and exhaustive in clarification? \\
D17: & Are you interested in teaching topics? \\
D18: & Are you overall satisfied with the teaching?
\end{tabular}

The score has a higher value going towards the center of the figure, i.e., the outermost circles of the figure from red to orange indicate criticality, while if the score is positioned from light yellow to intense green circles, it affirms the satisfaction of the student. According to this, a score equal to ten is the best on our scale. For example, the question D13 reports the satisfaction of students in using laboratories with a score of 8.7, and D18 highlights the satisfaction with the teaching lessons with a score of 8.7.

However, the students' satisfaction is also shown by the number of students developing their degree thesis in wireless standards topics under the supervision of the teacher of the Wireless Networks course. Moreover, the level of standards education achieved attending the course is confirmed by the increasing number of employed graduates having their first job in the telecom industry one year after graduation.

The first phase of the methodology provides a high level description of the considered standard describing the theoretical concepts, identifying the techniques employed, and the working parameters through the lecture periods. Standard documents are shown to students, and the teacher reads and discusses with them some particular specifications or parameters, e.g., of the 3GPP 36 series on LTE [13]. An extensive bibliography of books, e.g., [14-17], and articles, usually in English, are provided to support the students' learning, while the material provided directly by the teacher is in the mother tongue.

All the provided materials are available and loaded on the Modular Object-Oriented Dynamic Learning Environment (MOODLE) web platform used for the management of courses at the University. MOODLE can be used as a file repository, for e-learning, for transmitting communications to the students by email or chat, for publishing information about the course, lessons, to manage task/exercises, tests, and examinations. A web page for the Wireless Networks course is available in MOODLE for the students with their personal login access. A list of the Frequently Asked Questions (FAQ) starting from students' comments and clarification requests on parts of the course is also available on the platform, and this resource is of great help as well. The lessons are also provided by the e-learning mode of MOODLE, and on scheduled days, remote students can attend lessons and discuss with the teacher the material previously downloaded.

In the second phase, the design and implementation of a subsystem/system is undertaken according to the standard's specifications, identifying the functionalities (boxes, modules), interconnections, and working parameters, while in the third phase, tests of the implemented system in terms of functionality and performance are carried out by reporting reviews on the standard part implemented, the phases of the assigned project, and the realized tests. These activities are mainly developed in laboratories at the University or at telecom operators, as shown in the next Section 4.

Moreover, the activities in the laboratory allow cooperative learning among the students of a group to exchange problems and solutions for the given project. Collaborative learning [18] within a laboratory group is an effective method to achieve a solid background in the practical 
implementation of a complex system and mainly to learn how to work on a team, as required by several industrial tasks. Students that are more expert or Ph.D. students can act as group leaders and help the others. The students can have better relationships with their young colleagues to ask for clarifications, to understand their failures, and to propose corrective measures.

Another way to improve student's implementation skills is through a close collaboration with industries and telecom operators, which can be carried out with professional seminars, with visits to telecom infrastructure, and with participation in industrial projects during periods of internship. Several experts from industry and telecom operators have been invited to hold specific seminars in the course of Wireless Networks. For example, this academic year, a technician from a national telecom operator highlighted seminars on the signaling and mobility aspects of LTE and on hardware details for eNodeB and terminals, while a technical manager of a local Small and Medium Enterprise (SME) analyzed the security issues for WLAN and wireless sensor networks.

During scheduled visits, the students can access the infrastructure of the telecom operator, often for the first time. For example, when the hardware components of a macro radio base station with, e.g., dual band crosspolar antennas or a small radio base station were shown, the students' reaction was one of surprise because all the needed hardware was contained in one rack, while in students' imagination, more space was necessary.

The mandatory period of internship can be attended in telecom laboratories under the double supervision of University and industrial tutors. This is of particular interest for the students because they get in touch, often for the first time, with the professional world, use new tools to increase their own knowledge on system design compliant with wireless standards' specifications, and can have a practical vision of their future job in telecommunications.

\section{Laboratory Experiences for Telecommunications Standards' Education}

In this section, some project works implemented in the laboratories following the second and third phases of our methodology are shown.

\subsection{Experiences of Collaboration with the Telecom Operator}

The experience of collaboration with telecom providers is very important to teach the standards to students in a practical way. According to the proposed methodology, in the first phase, the teacher describes with the help of slides, e.g., the downlink and uplink logical, transport, and physical channels of the LTE system and their functionalities; then the students learn and memorize the names and their related use (often only for the examination). In the next phases, in a telecom laboratory, a telecom technician monitors the LTE network with probes installed at the eNodeB and uses traffic tools to show the radio access requirements, the flow of data, which channels are involved, and how they are used to manage access and to establish a connection from UE to the eNodeB/core network and vice versa. As a consequence, the use of practical instruments and tools can improve the learning of LTE channels. For example, the teacher can explain the Radio Resource Control (RRC) states and the impact on UE latency tied to the change of the connection states [19], as shown in Figure 2.

Instead, a traffic analyzer tool used by the telecom operator can highlight which connection or idle states are activated in succession, and from case-to-case, the latency times can be calculated directly as in Figure 3. The student is therefore more motivated to understand and learn about the problem with practice. 


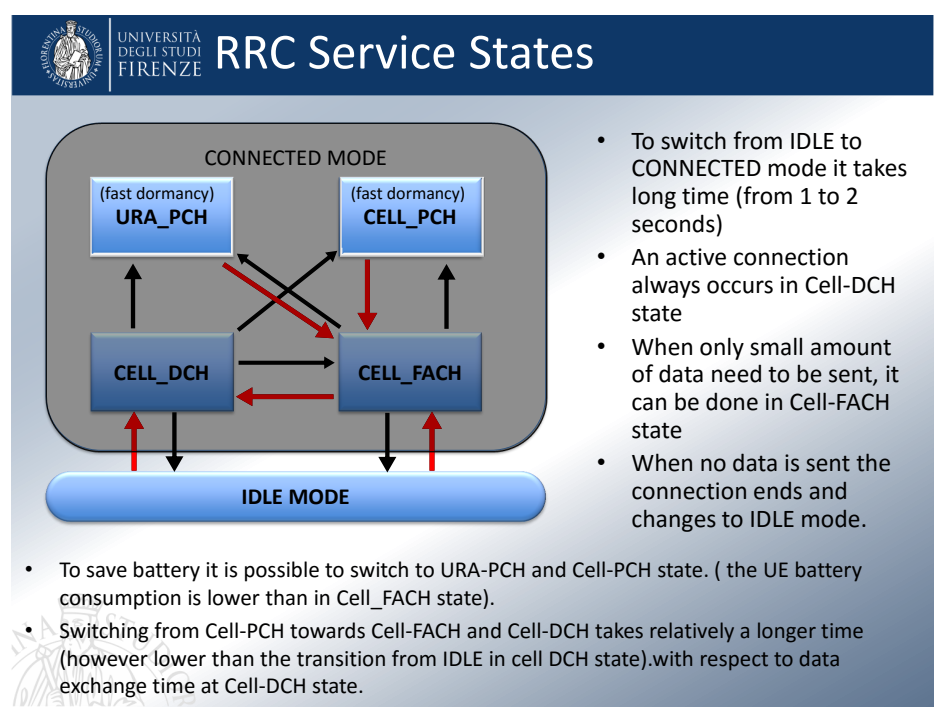

Figure 2. Radio Resource Control (RRC) states in the Universal Mobile Telecommunications System (UMTS) system.

Another work project allows the students to use the tools to monitor the traffic in the cells and analyze the different throughput in the downlink available according to the link adaptation concept, as in Figure 4. During the first phase, the students understand that the adaptation of modulation and coding to the link conditions is limited by the signal-to-noise plus the interference of other cells. In the second phase, the students can understand what good or bad channel conditions mean by analyzing the Signal-to-Interference-Noise Ratio (SINR) parameters shown in the tool.

Moreover, they can also learn the new concepts of Self-Organizing Networks (SON) introduced in 3GPP Release 9 for self-configuration, self-optimization, and self-healing of the network and analyze machine learning applications to optimize the management of specific Key Performance Indicators (KPIs) with respect to the users' satisfaction, i.e., the quality of experience [20-22].
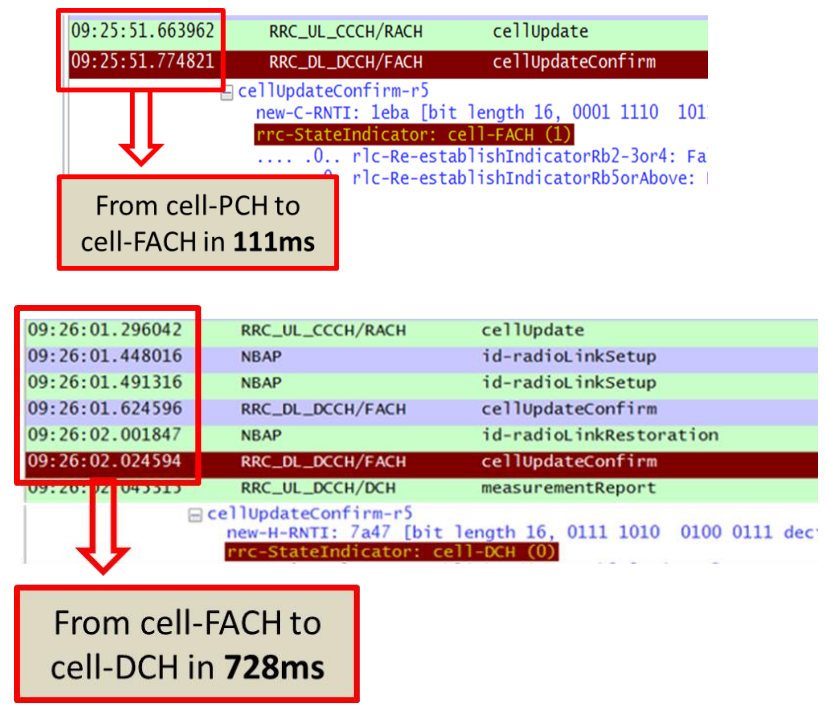

Figure 3. UE latency analysis shown by the telecom tool. 


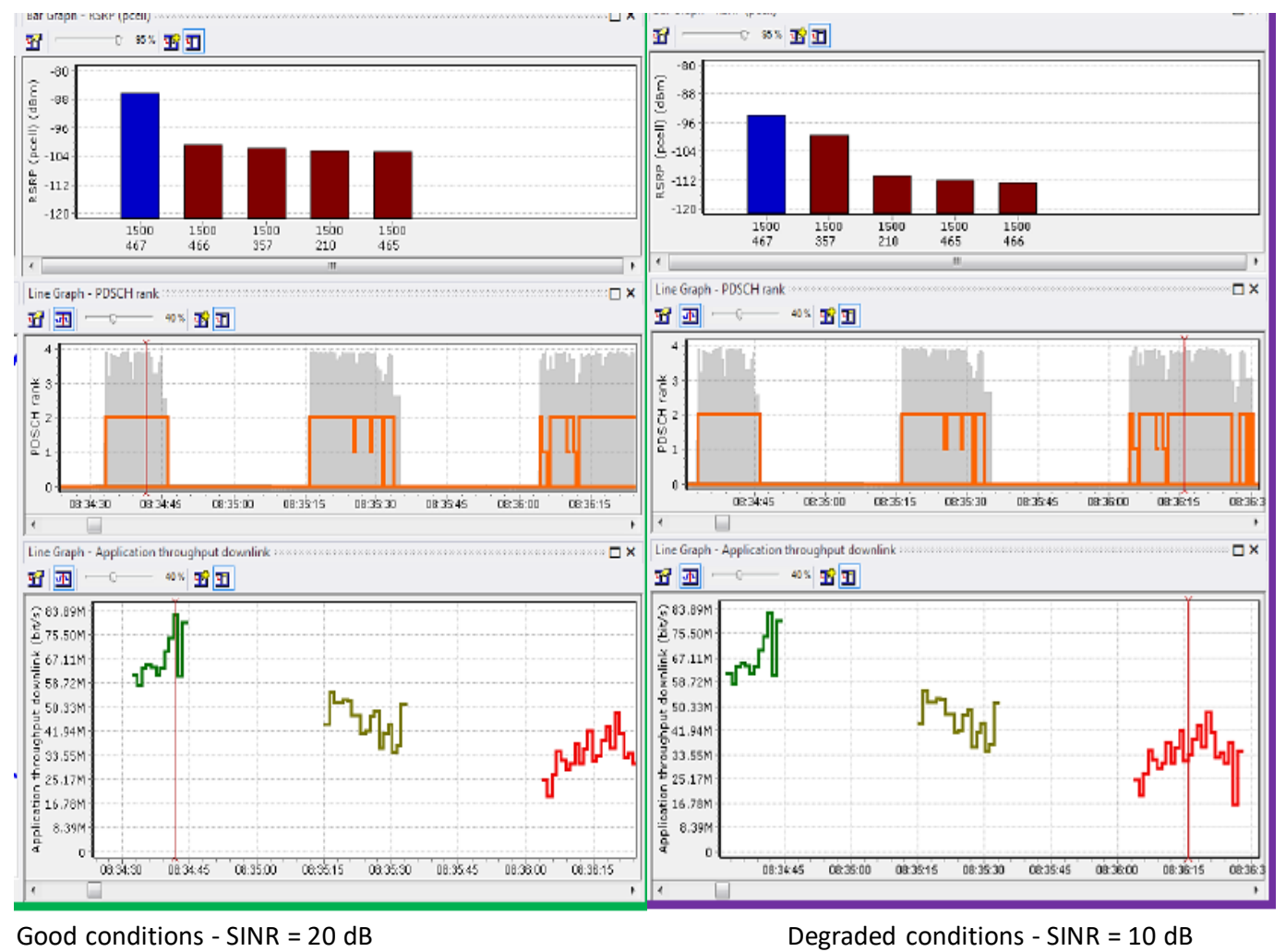

Figure 4. DL and UL throughput in different link conditions.

\subsection{Laboratory Experiences by Using MATLAB}

The University has a Total Academic Headcount license for MATLAB $\AA$ tools, a trademark of The MathWorks, Inc., used by professors and researchers for educational and research goals and by the students for the improvement of their knowledge in the telecom area. MATLAB offers many tutorials in the Communications Toolbox, which cover for example the physical layer aspects of the most common standards, UMTS, LTE, LTE-Advanced, and IEEE 802.11b. Moreover, many interesting simulation examples can be found on the site https://it.mathworks.com/matlabcentral developed by the user community for an open exchanging of MATLAB and Simulink files and models.

By using the MATLAB LTE System toolbox, it is easy to show the physical processing chain of LTE, e.g., time-frequency resources grid, explaining the position in the sub-carriers and slots of the main physical downlink channels, and the reference signals with different positions according to the multiple antenna scheme used.

A complete transmitter-channel-receiver chain can be simulated showing how to generate data as random bits, the effect of modulation, and the mapping to every symbol in a subframe. Then, the frame with primary and secondary synchronization signals and reference signals is formed, OFDM modulated, and passed through a fading channel with the addition of Additive White Gaussian Noise (AWGN). The students can understand the impact of the fading channel on the received grid, and different models of the fading channels are available in MATLAB with different delay profiles and related powers, for pedestrian, urban, vehicular, and MIMO propagation conditions compliant with 3GPP Series 36.

According to the methodology shown in Section 3.2, different projects can be developed by students to understand the LTE physical layer, e.g., the techniques that dynamically change transmission parameters with the varying channel conditions measured at the UE terminals, i.e., the link adaptation techniques. The Channel Quality Indicator (CQI), Precoder Matrix Indicator (PMI), and Rank Indicator (RI) measurements reported in the feedback for eNodeB allow dynamically changing the system parameters such as the system bandwidth, the MIMO transmission modes, 
the number of transmission layers, the precoding matrix, Modulation and Coding Schemes (MCSs), and the transmission power.

\subsection{Laboratory Experience for ZigBee Devices}

In the University laboratory, to understand wireless sensors networks and the Internet of Things environment practically, the students have available open source hardware and the OpenMote platform, with the OpenWSN OS, which integrates the OpenWSN protocol stack. The students can use the OpenMote platforms to learn, for example, how to compile the OpenMote firmware and how to use buttons or flashing LEDs. They need to use the Ubuntu operating system on the computer, program in $\mathrm{C} / \mathrm{C}++$ code for the ARM cortex platform, and in Python; therefore, they learn the operational aspects of a sensor network deployment. The devices are OpenMote CC2538, and in particular, CC2538 is an SoC (System on Chip) from Texas Instruments with a powerful wireless microcontroller system-on-chip; the radio operates at $2.4 \mathrm{GHz}$ and is fully compatible with the IEEE 802.15.4-2006 standard, 6LoWPAN, and ZigBee applications. For example, a prototype based on OpenMote-CC2538 has been implemented in the laboratory to highlight the security aspects for a wireless sensor network, for the $\mathrm{Wi}-\mathrm{Fi}$, or for the Ethernet connection, as shown in Figure 5.

A smart gateway is implemented on the UDOO Quad platform with installed in the open source Intrusion Detection System (IDS) (e.g., Snort). When it detects abnormal behavior by some device connected to the network (Wi-Fi or Ethernet connection or the wireless IoT links), it provides an alert. This is transmitted to a server, and in this case, a database (it is possible to use different solutions in the future, such as a server in the cloud) will provide to the user a simplified and interactive visualization of the alert through a graphical user friendly interface. Then, the necessary actions against the intrusion are implemented automatically, e.g., disconnecting the terminal or sensor from the network. Three types of attacks are simulated: a ransomware that tries to communicate with a command to a control center, a port scan attack, and an unauthorized access/query to the sensors via the Constrained Application Protocol (CoAP) [23].

By using this framework, the students can develop skills in wireless sensor networks, to connect devices with the gateway, learning to read the device hardware and firmware characteristics, and interrogate a sensor via the CoAP protocol. These hands-on experiences allow enlarging the technical knowledge and skills of students' approach to protocols and devices that they can use in their professional future.

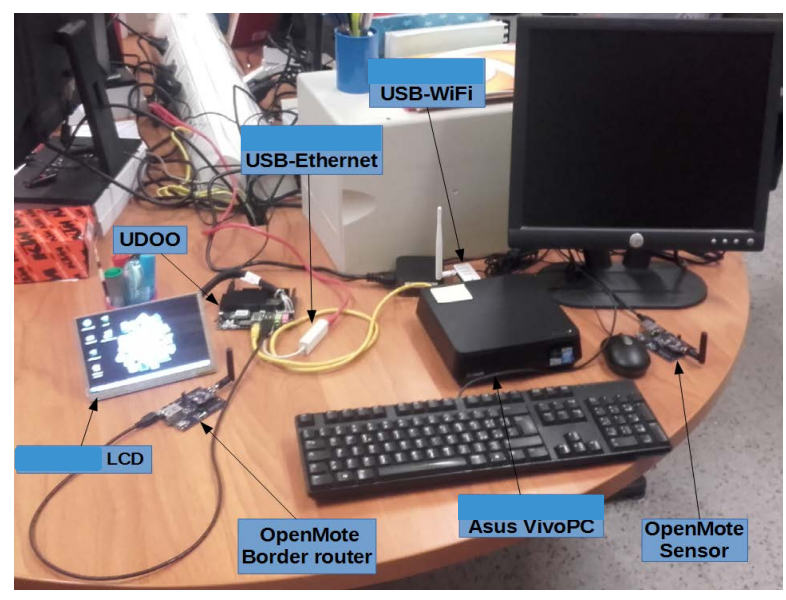

Figure 5. Wireless sensor network.

\section{Conclusions}

The paper highlighted the main problems and challenges in teaching wireless communication standards at the graduate level with the aim of updating the theoretical knowledge of students and 
training them with hands-on experiences for their future jobs in the wireless communications industry. The professor is the main actor in the process of translation from the technical language and contents of the standards to an easy standardization education for the students. The adopted methodology in the Wireless Networks course joins traditional lectures with practical activities in the laboratory to meet the objective of wireless standards' education according to the CDIO initiative and PBL.

The main contribution of the paper was to define a structured and sequential methodology for wireless standards teaching and to underline some study cases and laboratory projects to apply the methodology to the implemented standards compliant systems. Cooperative projects with telecom operators could show new practical horizons, providing valid contributions so that students can acquire skills in the implementation of wireless communications systems.

The students' satisfaction with the effectiveness of the adopted methodology of teaching was evaluated through questionnaires reporting an average score of eight in the range of [1-10], through the number of Master' degree theses developed on these topics, and through the number of students employed in telecom industries within one year after their graduation.

Funding: This research received no external funding

Acknowledgments: The author wishes to express her sincere appreciation to the telecom operators for the support in improving the standardization learning in the Wireless Networks course.

Conflicts of Interest: The author declares no conflict of interest.

\section{References}

1. Demestichas, P.; Georgakopoulos, A.; Karvounas, D.; Tsagkaris, K.; Stavroulaki, V.; Lu, J.; Xiong, C.; Yao, J. 5G on the Horizon: Key Challenges for the Radio-Access Network. Veh. Technol. Mag. IEEE 2013, 8, 47-53. [CrossRef]

2. Boccardi, F.; Heath, R.; Lozano, A.; Marzetta, T.; Popovski, P. Five disruptive technology directions for 5G. IEEE Commun. Mag. 2014, 52, 74-80. [CrossRef]

3. Pierucci, L. The quality of experience perspective toward 5G technology. IEEE Wirel. Commun. 2015, 22, 10-16. [CrossRef]

4. Katusic, D.; Skocir, P.; Kusek, M.; Jezic, G.; Ratti, C.; Bojic, I. Hands-On Education about Standardization: Is That What Industry Expects? IEEE Commun. Mag. 2017, 55, 133-144. [CrossRef]

5. Gelonch-Bosch, A.; Marojevic, V.; Gomez, I. Teaching Telecommunication Standards: Bridging the Gap between Theory and Practice. IEEE Commun. Mag. 2017, 55, 145-153. [CrossRef]

6. Available online: http:www.cdio.org (accessed on 13 December 2019).

7. Edström, K.; Kolmos, A. PBL and CDIO: Complementary models for engineering education development. Eur. J. Eng. Educ. 2014, 39, 539-555. [CrossRef]

8. Hassan, O.A. Learning theories and assessment methodologies-An engineering educational perspective. Eur. J. Eng. Educ. 2011, 36, 327-339. [CrossRef]

9. Biggs, J.; Tang, C. Teaching For Quality Learning At University; Society for Research into Higher Education and Open University Press Imprint, McGraw-Hill Education: London, UK, 2011.

10. Developing Graduate Engineering Skills. Available online: http://sefibenvwh.cluster023.hosting.ovh.net/ wp-content/uploads/2017/07/POsition-Paper-on-Engineering-Skills.pdf (accessed on 13 December 2019).

11. Chenard, J.; Zilic, Z.; Prokic, M. A Laboratory Setup and Teaching Methodology for Wireless and Mobile Embedded Systems. IEEE Trans. Educ. 2008, 51, 378-384. [CrossRef]

12. Available online: http:http://valmon.disia.unifi.it/sisvaldidat/unifi/ (accessed on 13 December 2019).

13. Available online: https://www.3gpp.org/DynaReport/36-series.htm (accessed on 13 December 2019).

14. Molish, A. Wireless Communications; John Wiley and Sons Ltd. Publishing: Chichester, UK, 2012.

15. Stallings, W. Wireless Communications and Networks, 1st ed.; Pearson Prentice Hall: Upper SaddleRiver, NJ, USA, 2005; p. 07458.

16. Holma, H.; Toskala, A. LTE for UMTS: Evolution to LTE-Advanced, 2nd ed.; John Wiley and Sons Publishing: Chichester, UK, 2011.

17. Khan, F. LTE for 4G Mobile Broadband: Air Interface Technologies and Performance, 1st ed.; Cambridge University Press: New York, NY, USA, 2009. 
18. Hamid, R.; Baharom, S.; Taha, M.R.; Kadaruddin, L.K. Competition as an Innovative Student-centered Learning Method for Open-ended Laboratory Work. Procedia-Soc. Behav. Sci. 2013, 102, 148-152. [CrossRef]

19. Micheli, D.; Curto, A.; Mazzurco, M.; Pierucci, L.; Fantacci, R.; Simoni, R. Handset and network quality performance benchmarking for QoE improvement. In Proceedings of the 2013 IEEE Globecom Workshops (GC Wkshps), Atlanta, GA, USA, 9-13 December 2013; pp. 1145-1150. [CrossRef]

20. Pierucci, L.; Romoli, A.; Fantacci, R.; Micheli, D. An optimized neural network for monitoring Key Performance Indicators in HSDPA. In Proceedings of the 21st Annual IEEE International Symposium on Personal, Indoor and Mobile Radio Communications, Istanbul, Turkey, 26-29 September 2010; pp. 2041-2045. [CrossRef]

21. Pierucci, L.; Micheli, D. A Neural Network for Quality of Experience Estimation in Mobile Communications. IEEE MultiMedia 2016, 23, 42-49. [CrossRef]

22. Chiti, F.; Pierucci, L. API2T: A bit of improvement for applications in critical scenarios. In Proceedings of the 6th International Wireless Communications and Mobile Computing Conference (IWCMC '10), Caen, France, 28 June-2 July 2010; pp. 794-798. [CrossRef]

23. Pecorella, T.; Pierucci, L.; Nizzi, F. "Network Sentiment" Framework to Improve Security and Privacy for Smart Home. Future Internet 2018, 10, 125. [CrossRef]

(C) 2019 by the author. Licensee MDPI, Basel, Switzerland. This article is an open access article distributed under the terms and conditions of the Creative Commons Attribution (CC BY) license (http://creativecommons.org/licenses/by/4.0/). 